\title{
Comment on "Preoperative anxiety induces chronic postoperative pain by activating astrocytes in the anterior cingulate cortex region"
}

\author{
(iD) Yu Zhong ${ }^{1}$ \\ (iD) Qiong Zhang ${ }^{2}$ \\ (DD Zhaoyang Zeng' \\ (D) Wenrong Huang ${ }^{1}$ \\ (D) Zhiwei Huang ${ }^{1}$ \\ (i) Xing Chen ${ }^{1}$
}

1. Department of Endocrinology, the First College of 'YYClinical Medical Science, China Three Gorges University, Yichang Central People's Hospital, Yichang, Hubei 443003, China. 2. Department of Anesthesiology, The People's Hospital of China Three Gorges University, The First People's Hospital of Yichang, Yichang, Hubei 443000, China.

Dear Editor,

We read with great interest the study by Gu et al. ${ }^{1}$ in which they demonstrated that anxiety plays a key role in the development of chronic pain. This study offers new guidance for improving chronic postoperative pain. However, some concerns should be raised.

To begin with, we support the comments by Xue et al. ${ }^{2}$ that more methods for the model of anxiety were necessary. Additionally, it is also important for a clinical study to explore the relationship between anxiety and the risk of chronic postoperative pain. Nerve injury and inflammation promote chronic postoperative pain. Thus, the authors could determine the inflammation response of the mouse. Single-prolonged stress modeling and hysterectomy should be used as a reference. The definition of a successful model of single-prolonged stress should be addressed in detail.

Secondly, more experiments were needed to confirm the relationship between the activation of astrocytes and chronic pain. The activation of astrocytes may be the result of chronic pain but not the cause. Thus, improving the lifestyle may be an effective way to reduce the duration of chronic postoperative pain.

\section{REFERENCES}

1. Gu D, Zhou M, Han C, Lei D, Xie S, Yuan Y, et al. Preoperative anxiety induces chronic postoperative pain by activating astrocytes in the anterior cingulate cortex region. Rev Assoc Med Bras. 2019;65(9):1174-80.

2. Xue B, He L. Comments: "Preoperative anxiety induces chronic postoperative pain by activating astrocytes in the anterior cingulate cortex region". Rev Assoc Med Bras. 2019;65(9):1181. 